\title{
Erratum
}

\section{The Radon Transform for Forms of Type $(1,1)$ on Complex Projective Space}

Math. Ann. 271, 485-492 (1985)

\section{Hubert Goldschmidt}

Department of Mathematics, Columbia University, New York, NY 10027, USA

Propositions 1 and 2 are true only for $n=2$. If $n \geqq 3$ and $\gamma$ is the element $(q+1) \lambda_{0}$ $+\lambda_{1}-\lambda_{n-1}-(q+1) \lambda_{n}$ of $\hat{G}$, with $q \geqq 0$, the multiplicity of $C_{\gamma}^{\infty}\left(T^{1,1}\right)$ is equal to one (see [8, Proposition 3.4]). Consequently, Theorem 1 holds only when $n=2$, and is trivial in this case. The proof of Theorem 2 remains valid when $n=2$. Based on joint work with Jacques Gasqui, we now proceed to give a proof of Theorem 2 for all dimensions $n \geqq 2$, and to generalize this result to forms of type $(p, p)$.

Let $X$ be a compact Kähler manifold of dimension $n$. Let $T^{\prime}$ be the bundle of holomorphic tangent vectors. Let $\mathscr{A}^{p}$ be the space of complex-valued forms of degree $p$ on $X$. We write $\mathscr{A}^{p, q}=C^{\infty}\left(T^{p, q}\right)$ and let $\pi_{p, q}$ be the natural projection of $\mathscr{A}^{p+q}$ onto its subspace $\mathscr{A}^{p, q}$. Let $\mathbf{H}^{p, q}$ denote the space of harmonic forms of type $(n, q)$.

Standard Hodge theory gives us the closed orthogonal decomposition

$$
\begin{aligned}
\mathscr{A}^{p, q}= & \mathbf{H}^{p, q} \oplus \partial \bar{\partial} \mathscr{A}^{p-1, q-1} \oplus \partial \bar{\partial}^{*} \mathscr{A}^{p-1, q+1} \\
& \oplus \bar{\partial} \partial^{*} \mathscr{A}^{p+1, q-1} \oplus \partial^{*} \bar{\partial}^{*} \mathscr{A}^{p+1, q+1}
\end{aligned}
$$

from which we immediately deduce

lemma A. If $\alpha \in \mathscr{A}^{p, q}$ satisfies $\partial \bar{\partial} \alpha=0$, then there exist $\varphi \in \mathbf{H}^{p, q}, \beta_{1} \in \mathscr{A}^{p-1, q}$, $f_{2} \in \mathscr{A}^{p, q-1}$ such that

$$
\alpha=\varphi+\partial \beta_{1}+\delta \beta_{2} .
$$

Let $\omega^{p}$ denote the $p^{\text {th }}$ exterior power of $\omega$ and $\left\{\omega^{p}\right\}$ the sub-bundle of $T^{p, p}$ generated by its section $\omega^{p}$. If $\Lambda: T^{p, q} \rightarrow T^{p-1, q-1}$ is the trace morphism induced by interior multiplication by $\omega$, from the standard formulas

$$
\bar{\partial}^{*}=i(\Lambda \partial-\partial \Lambda), \quad \partial^{*}=-i(\Lambda \bar{\partial}-\bar{\partial} \Lambda),
$$

there are constants $c_{1}, c_{2}$ such that

$$
\bar{\partial}^{*} \partial^{*}\left(f \omega^{p}\right)=c_{1}(\Delta f) \omega^{p}+c_{2} \partial \bar{\partial} f \wedge \omega^{p-1},
$$

for all $f \in C^{\infty}(X)$. Since $T^{n, n}$ is generated by its section $\omega^{n}$, we obtain the inclusion

$$
\partial^{*} \bar{\partial}^{*} \mathscr{A}^{n, n} C C^{\infty}\left(\left\{\omega^{n-1}\right\}\right)+\partial \check{\partial} C^{\infty}\left(\left\{\omega^{n-2}\right\}\right) \text {. }
$$


If $\mathbf{H}_{0}^{p, p}$ denotes the subspace of $\mathbf{H}^{p, p}$ of harmonic forms of type $(p, p)$ whose trace vanishes, (1) and (2) give us:

Lemma B. We have the decomposition

$$
\begin{aligned}
\mathscr{A}^{n-1, n-1}= & \left(\mathbf{H}_{0}^{n-1, n-1} \oplus \partial \bar{\partial} \mathscr{A}^{n-2, n-2} \oplus \partial \bar{\partial}^{*} \mathscr{A}^{n-2, n}\right. \\
& \left.\oplus \bar{\partial} \partial^{*} \mathscr{A}^{n, n-2}\right)+C^{\infty}\left(\left\{\omega^{n-1}\right\}\right) .
\end{aligned}
$$

When $X=\mathbf{C P}^{2}$, we recover the result of Proposition 2.

The proof of the following lemma is elementary and is left to the reader.

Lemma C. Let $\alpha \in T^{p, p}$, with $p \geqq 1$. If

$$
\alpha\left(\xi_{1}, \ldots, \xi_{p}, \bar{\xi}_{1}, \ldots, \bar{\xi}_{p}\right)=0,
$$

for all $\xi_{1}, \ldots, \xi_{p} \in T^{\prime}$, then $\alpha$ vanishes.

For $p \geqq 1$, we consider the first-order differential operator

$$
D=\pi_{p, p} d: \mathscr{A}^{2 p-1} \rightarrow \mathscr{A}^{p, p} .
$$

If $\alpha \in \mathscr{A}^{2 p-1}$, we have

$$
D \alpha=\bar{\partial} \pi_{p, p-1} \alpha+\partial \pi_{p-1, p} \alpha
$$

and

$$
\partial \bar{\partial} D=0 ;
$$

moreover, the integral of $D \alpha$ vanishes over an arbitrary closed complex submanifold of $X$ of dimension $p$.

We now suppose that $X=\mathbf{C P}^{n}$. From the preceding lemma and the description of the tangent spaces of the linearly imbedded complex projective $p$-planes in $\mathbf{C P}^{n}$ given by $[1$, p. 79$]$, we obtain:

Lemma D. Let $X=\mathrm{CP}^{n}$ and $\alpha$ be an element of $\mathscr{A}^{k, k}$. The following assertions are equivalent:

(i) $\partial \bar{\partial} \alpha=0$;

(ii) for every complex projective $(k+1)$-plane $Y$ of $\mathbf{C P}^{n}$, the restriction $\alpha_{\mid Y}$ of $\alpha$ to $Y$ satisfies $\partial \bar{\partial}\left(\alpha_{\mid Y}\right)=0$.

The following result is a generalization of Theorem 1 .

Theorem. Let $X=\mathbf{C P}^{n}$ and $\alpha \in \mathscr{A}^{k, k}$, with $n \geqq 2$ and $1 \leqq k \leqq n-1$. Then the following statements are equivalent:

(i) The integral of $\alpha$ over every complex projective $k$-plane of $\mathbf{C P}^{n}$ vanishes;

(ii) There exists an element $\beta$ of $\mathscr{A}^{2 k-1}$ such that $D \beta=\alpha$.

Proof. It suffices to prove (i) $\Rightarrow$ (ii). Assume that (i) holds. Let $Y$ be a complex projective $(k+1)$-plane of $\mathbf{C P}^{n}$ and $\omega_{Y}$ be its Kähler form. According to Lemma B, we may write

$$
\alpha_{\mid Y}=f \omega_{Y}^{k}+D \varphi
$$


where $f$ is a complex-valued function and $\beta$ is a form of degree $2 k-1$ on $Y$. Condition (i) implies that the integral of $f$ vanishes over every complex projective $k$-plane of $Y$. Thus, by Proposition 3, (i), $f$ vanishes, and so $\partial \bar{\partial}\left(\alpha_{\mid Y}\right)=0$. From Lemmas $A$ and $D$, we infer that

$$
\alpha=c \omega^{k}+D \beta,
$$

where $c \in \mathbf{C}$ and $\beta \in \mathscr{A}^{2 k-1}$. By condition (i), the constant $c$ must be equal to zero.

\section{References (continued)}

8. Tsukamoto, C.: Infinitesimal Blaschke conjectures on projective spaces. Ann. Sci. Éc. Norm. Super., IV. Ser. 14, 339-356 (1981)

Received September 6, 1985 